\title{
PRC1 Gene
}

National Cancer Institute

\section{Source}

National Cancer Institute. PRC1 Gene. NCI Thesaurus. Code C118349.

This gene is involved in cytokinesis. 